\title{
Synaptic development and branch dynamics
}

DOI:

10.1038/nrn1921
The development and refinement of topographic maps in the brain depends on dynamic changes in the precise arrangement of axonal branches. Two articles in The Journal of Neuroscience provide a window onto the growth of presynaptic axonal branches during the early development of living retinal ganglion cells. Both studies report that synaptogenesis and synaptic maturation make important contributions to the growth and stabilization of axonal branches.

In these two studies, the authors tracked over time the effects of synaptic development on axonal branch dynamics in retinal ganglion cells projecting from the retina to the optic tectum in the midbrain. They used two-photon time-lapse imaging of the GFP (green fluorescent protein)-labelled synaptic vesicle protein synaptophysin. This was co-expressed with cytosolic dsRED (discosoma red fluorescent protein) to mark arbor structure. They then quantified the levels of synaptophysin at individual synapses to provide a measure of the extent of maturation or disassembly of synapses over time, and related this to structural changes in axonal arbors.

In their study of retinal ganglion cells of albino Xenopus laevis tadpoles, Ruthazer and colleagues found that presynaptic sites that were intensely labelled with GFP-tagged synaptophysin had more presynaptic vesicles

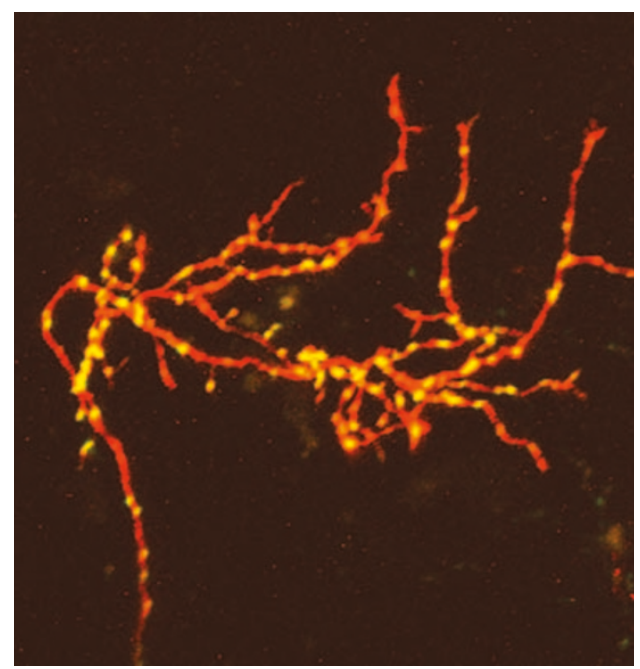

A Xenopus retinal ganglion cell axon expressing dsRED and GFP-tagged synaptophysin imaged in vivo. Image courtesy of H. Cline, Cold Spring Harbor Laboratory, USA, and E. Ruthazer, Montreal Neurological Institute, Canada.

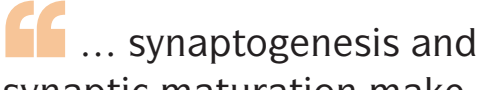
synaptic maturation make important contributions to the growth and stabilization of axonal branches.

and were more stable. Synapses were the sites of new branch additions, so that axon arbor development was clearly affected by synapse formation and stabilization. Ruthazer and colleagues were particularly interested in branch elimination and observed that strong synapses stabilized branches against retraction. Meyer and Smith confirmed this close association between the timecourse of synaptic development and the growth of axonal arbors in a study of retinal ganglion cells in living zebrafish larvae.

Through careful analysis of changes in levels of synaptophysin over time, and branch extension and lifetime, both sets of authors showed that the presence of stabilized synaptic contacts directly influenced the extent to which branches stabilized, rather than being a consequence of branch stabilization. These data suggest a model in which synaptogenesis and synaptic maturation influence the growth of axonal arbors by stimulating the formation and extension of new branches, and by selectively stabilizing them.

Ruthazer and colleagues also showed that visual stimulation enhanced the stability of arbors that contained more maturing synapses, whereas it induced retraction of newly formed, exploratory branch tips.

These two studies provide firm evidence for the participation of synaptic development in the shaping of topographic maps, and shed light on the mechanisms involved in this process.

\section{Alison Rowan}

ORIGINAL RESEARCH PAPERS Ruthazer, E. S., Li, J. \& Cline, H. T. Stabilization of axon branch dynamics by synaptic maturation. J. Neurosci. 26, 3594-3603 (2006) | Meyer, M. P. \& Smith, S. J. Evidence from in vivo imaging that synaptogenesis guides the growth and branching of axonal arbors by two distinct mechanisms. J. Neurosci. 26, 3604-3614 (2006) FURTHER READING Innocenti, G. M. \& Price, D. J. Exuberance in the development of cortical circuits. Nature Rev. Neurosci. 6. 995-965 (2005) 